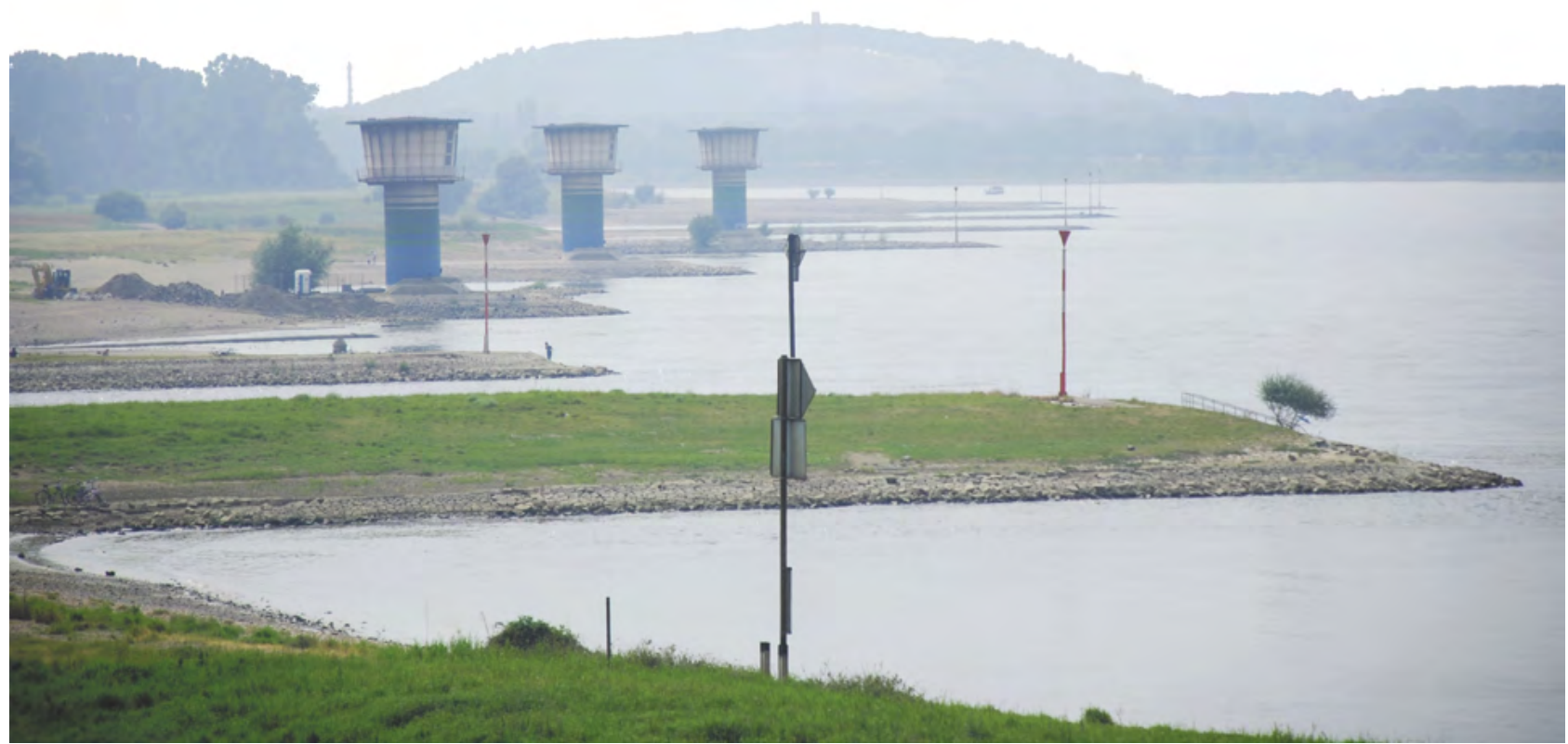

Faculté d'architecture, d'ingénierie architecturale, d'urbanisme de l'Université catholique de Louvain 


\title{
Entretien avec Andreas Hofer : Du commun à la ville
}

\author{
Chloé Salembier, Anna Ternon et Marine Declève
}

Andreas Hofer travaille comme architecte et urbaniste. Il a fondé en 1995 avec sespartenaires le bureau d'architecture Archipel à Zurich et a lancé en 1993 le projet de coopérative de logement Kraftwerk 1. Il soutient aujourd' hui le développement de nouveaux projets de coopératives de logement. Depuis 2008, il est responsable du montage du projet expérimental Mehr als wohnen à Zurich. Andreas Hofer enseigne régulièrement dans différentes universités, il est membre de jurys de concours d'architecture et publie des textes sur le développement de la ville, la conversion des friches industrielles et les nouvelles typologies de logement répondant aux défis d'une société postindustrielle en transformation.

Lors de sa conférence « Du commun à la ville » à LOCI Saint-Gilles, Andreas Hofer nous accorde un entretien dans le cadre du premier Massive Open Online Class (MOOC) de la faculté portant sur les récits d'urbanisme et la question des communs. Dans cet entretien, il expose l'évolution historique du concept depuis la notion de allmende (communs) jusqu'à la redéfinition des coopératives de logement à partir des années 1980 dans le contexte de la confédération helvétique. Il revient sur la transformation de son rôle d'architecte dans le développement de projets de coopératives et sur la philosophie qui se trouve au cour des projets Kraftwerk 1 (1993) et Mehr als wohnen (2007).

Pourrais-tu te présenter et nous expliquer en quelques mots comment tu en es arrivé à travailler sur la question des coopératives?

J'ai étudié l'architecture à l'École Technique de Zurich (ETH Zurich) dans les année 1980. À cette époque, la ville connaissait une grande pénurie du logement, beaucoup de manifestations et de conflits sur cette question. Nous nous sommes sentis concernés en tant que professionnels et cela nous a lié à des discussions politiques, des protestations, des squats... De ce conflit dans les années 1990 est né un nouveau mouvement de coopératives qui a essayé de reprendre cette structure organisatrice en la remplissant avec les nouveaux thèmes qui nous occupaient à l'époque : l'intégration, le logement abordable et l'environnement. Ces projets ont connu un assez grand succès, et ça m'a conduit à transformer ma profession, maintenant je développe de tels projets. Je ne suis plus seul, il y a aujourd'hui beaucoup de gens qui se préoccupent de ce thème. II y a aussi une nouvelle génération qui assure la transition. C'est un moment intéressant parce que ce ne sont plus des petits projets pionniers mais c'est vraiment un mouvement qui change et qui transforme la ville.

Pourquoi y a-t-il eu ce renouveau des coopératives de logement en Suisse ? Comment expliquer cette différence entre la Suisse et d'autres pays européens?

L'histoire du mouvement des coopératives à Zurich, c'est une histoire longue qui a débutée il y a une centaine d'années. Initialement, c'était lié à la démocratie sociale, aux mouvements des travailleurs venus travailler en ville. La

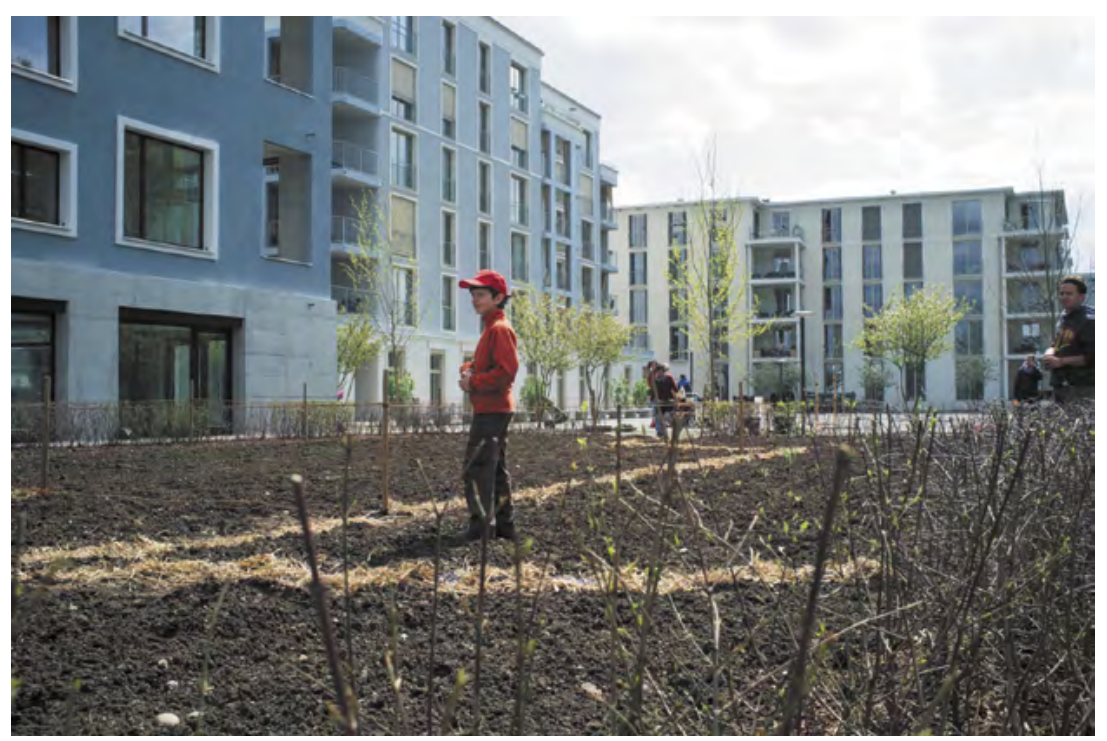




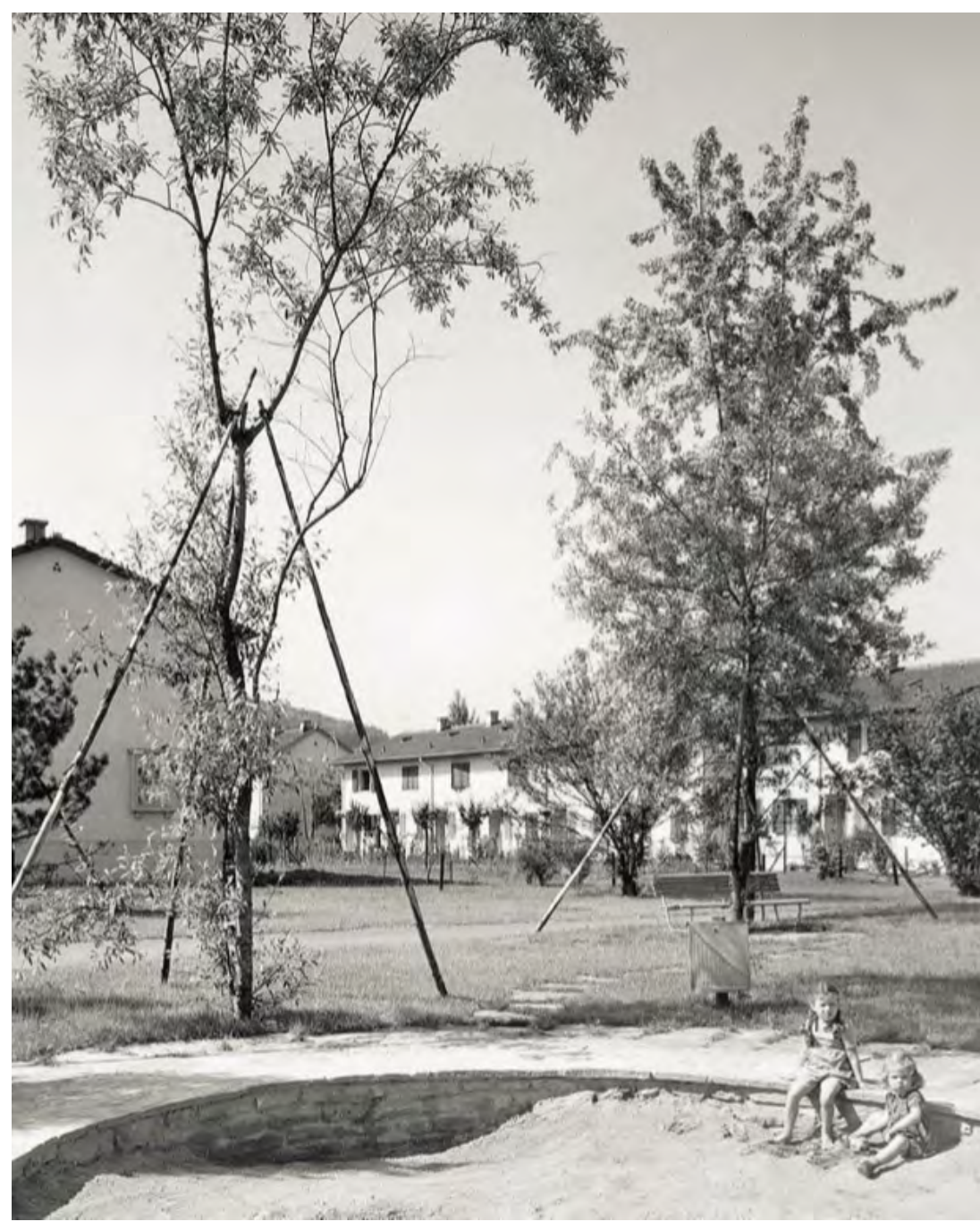

spécificité du système à Zurich, c'est qu'ils étaient invités par la ville à s'entraider. La ville a supporté les coopératives, mais elles ont gardé une indépendance très importante. Cela a permis de produire 40000 logements, c'est-à-dire $20 \%$ de l'ensemble des logements zurichois. Ces logements ont été construits par 120 coopératives, entre les années 1920 et 1970 . Ensuite, les coopératives ont cessé de se développer. Dans les années 1970-1980 avec le processus de désindustrialisation de la Suisse, la ville a sombré dans une forte crise et a perdu $20 \%$ de ses habitants. Les années 1980-1990 c'était vraiment, une baisse, une crise, il manquait d'idées. Nous avons pris l'initiative de rependre les structures anciennes pour les remplir avec des idées nouvelles. Ça a marché et aujourd'hui nous sommes dans un processus où les énormes terrains, les bâtiments existants des anciennes coopératives sont aussi à disposition pour être rénovés ou remplacés. Ce contexte crée une atmosphère assez collaborative entre les anciennes coopératives qui cherchent un futur et les jeunes pionniers qui n'ont pas les terrains. Aujourd'hui, nous travaillons vraiment en- semble. Le projet Mehr als wohnen (plus qu'habiter) est le symbole de cette collaboration parce que cette coopérative a été fondée par plus d'une trentaine des coopératives existantes, très traditionnelles, mais aussi avec des pionniers. On a essayé de discuter ensemble et finalement cela nous a amené à construire presque 400 logements.

Pourrais-tu expliquer le projet de Mehr als wohnen ? Quel est le processus de production de ces logements ? Quel est le modèle de gouvernance que vous mettez en place pour ce type de projet?

Mehr als wohnen est née d'un anniversaire, on a fêté en 2007 les cent ans de la loi de 1907 sur le logement d'utilité publique à Zurich. Lors de ce centenaire - que j'ai organisé comme représentant de l'association des coopératives zurichoises - on a beaucoup discuté. C'était vraiment une phase de renaissance qui a permis de repenser le thème du développement de la ville. De ces discussions est née l'idée de prouver à l'échelle $1 / 1$ ce que nous pouvions faire comme expérimentation et on a proposé 
de fonder cette coopérative. Nous avons aussi discuté avec la ville de Zurich de ce que nous pouvions faire sur le terrain. Finalement nous avons fondé dans la même année du centenaire cette coopérative qui initie un modèle expérimental permettant d'apprendre à développer de nouvelles approches du logement. Nous avons organisé beaucoup de séances publiques où les coopératives traditionnelles sont venues pour apprendre à organiser une compétition architecturale, à discuter des thèmes liés à la durabilité... Ces aspects étaient très importants parce que certaines anciennes coopératives n'avaient pas construit pendant une quarantaine d'année. Nous avons aussi publié des articles sur la production de logement participatif : travailler ensemble, discuter ensemble, d'apprendre ensemble... C'était naturellement aussi un projet très discursif, nous avons fait beaucoup de séances publiques, de recherches et nous avons invité tout le monde à participer à ce processus. C'était des séances qui rassemblaient entre 200 et 300 personnes et qui ont vraiment permis de traiter les thèmes pertinents pour ce projet. Nous avons travaillé ensemble pendant huit ans.
Comment sont prises les décisions dans le cadre de ces processus participatifs?

Le système participatif a évolué, depuis le mouvement politique des années 1990. Nous n'avons jamais construit pour nous-même... ce n'était pas le thème, comme en Allemagne par exemple, où on a les bouwgroepen (groupes de construction), des gens qui veulent vivre ensemble, et qui s'organisent, et finalement ils construisent une quinzaine d'appartements dans lesquels ils habitent. Ça, nous n'avons jamais fait. Nous avons toujours voulu des relations à la politique de la ville. Nous avons donc développé des projets exemplaires pour démontrer comment les gens peuvent vivre ensemble. Ça n'a jamais été des projets exclusifs socialement. Ce n'est pas la couche moyenne, les professeurs par exemple, qui formeraient ensemble une nouvelle coopérative, non. Mehr als wohnen est situé dans le quartier le plus pauvre de Zurich et le projet renferme une dimension très intégrante. Mehr als wohnen, c'est 1200 habitants et 65 nations, des gens assez pauvres et des gens un peu plus influents. Ces derniers sont peut-être plus actifs dans

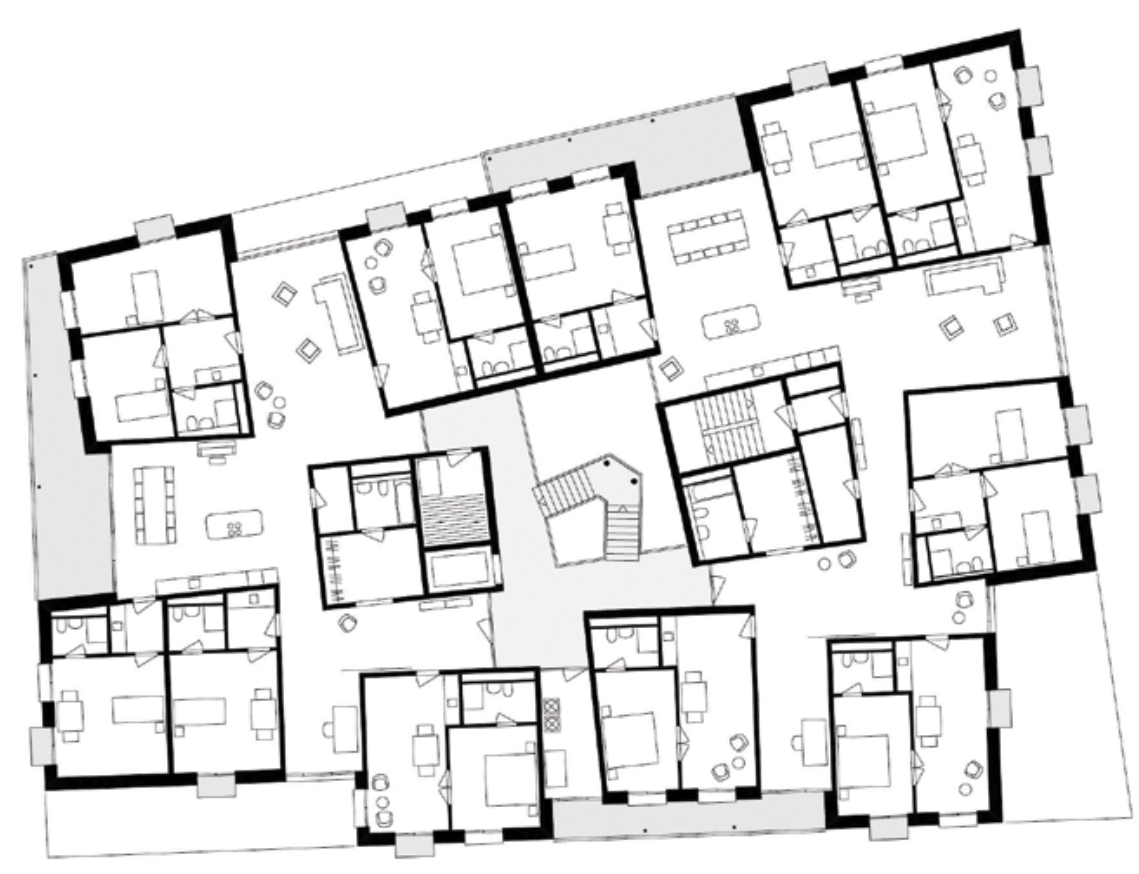




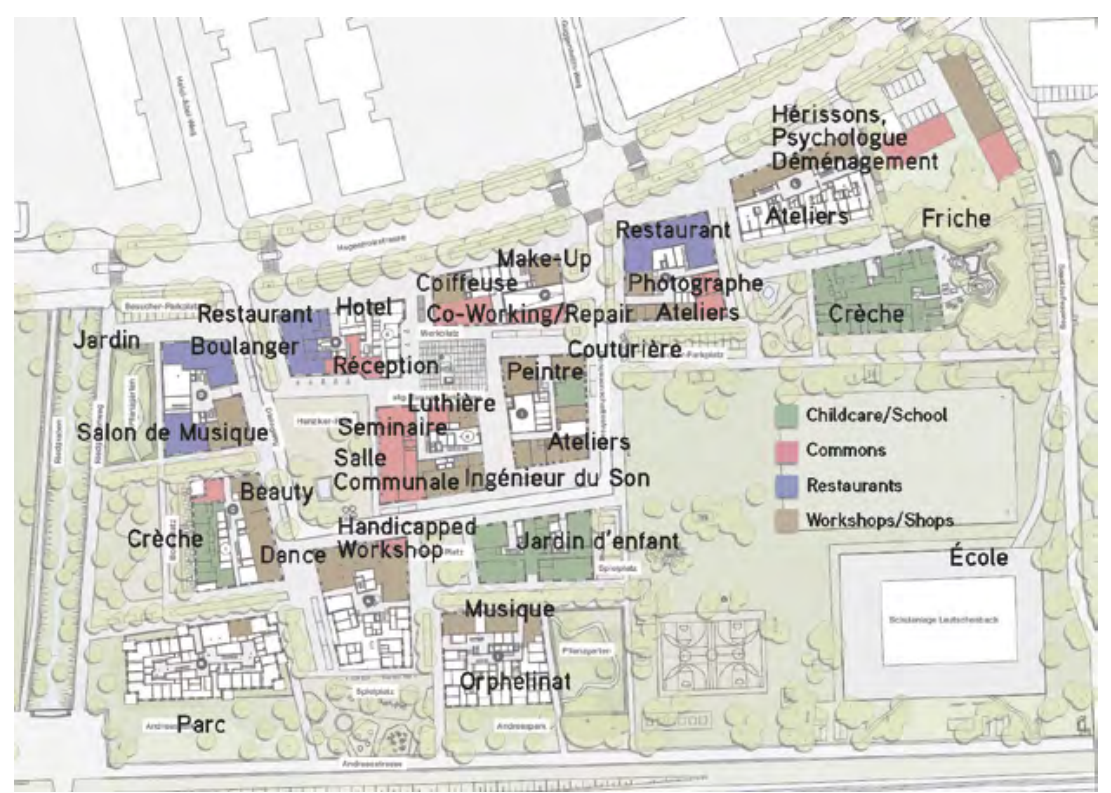

les processus participatifs. On ne peut pas réunir 1200 habitants qui discutent ensemble, mais on discute le quartier, on discute avec les professionnels. Nous avons construit avec cinq bureaux d'architectes, c'était aussi un processus participatif entre eux. On a des principes d'ouverture, toutes les séances sont publiques par exemples. Même les séances de jurys de la compétition étaient des événements publics et ça a créé une atmosphère on pourrait dire d'université populaire. On se rassemblait les soirs, on discutait, on faisait des groupes de travail pour certains thèmes... parce que nous avions l'habitude, ce n'était pas une première. II y a une chaine de projets en projets, c'est un processus qui se situe à l'échelle urbaine où nous essayons d'apprendre et de transformer la ville.

\section{À Zurich, il est possible d'être coopé- rant sans forcément habiter dans la coopérative. Comment fonctionne ce système?}

La grande majorité sont des coopératives de locataires. Les gens qui habitent là, ils doivent être coopérateurs. Mais parce que les projets sont aussi des projets de démonstration et d'expérimentation, il y a toujours eu des gens qui ont supporté le projet et qui sont devenus à leur tour coopérateurs sans nécessairement vouloir louer un appartement. Naturellement, derrière ça il y a aussi une raison financière. Moi par exemple je suis membre de 5 ou 6 coopératives. Si une nouvelle coopérative est fondée, ils lancent un fundraising, ils demandent publiquement pour être supportés. C'est un engagement politique de devenir membre de ces coopératives.
Quand tu dis que les coopératives de logement sont des projets pilotes, des projets qui expérimentent, qui développent une autre manière de penser la ville, qu'est-ce qui est vraiment innovant à Mehr als wohnen ?

La tradition des coopératives est née de la volonté de produire des appartements dans des lotissements monofonctionnels pour les familles traditionnelles qui avait des difficultés sur le marché du logement. On a fait ça pendant une centaine d'années. II y avait aussi une idée anticapitaliste. Les privés organisaient les fabriques, les commerces, et les coopératives organisaient le logement. C'est aussi lié au modernisme, qui a promu la séparation des fonctions. On a aussi essayé de lier les travailleurs dans la ville de Zurich. C'était une ville très industrielle, ce sont les paysans qui ont travaillé dans les fabriques. C'étaient des paysans pauvres qui sont venus des montagnes, dans la ville. On a essayé de les lier avec les lieux qu'ils ont quittés, en faisant des petites maisons homogènes, avec des petits jardins, ils ont eu des animaux aussi dans leur jardin. On a fait des images sur les murs, c'était toujours des paysans dans les champs... c'était toujours une schizophrénie culturelle pour accueillir les gens en ville. Ce système fordiste, pseudo rural, s'est complètement brisé. À Zurich, maintenant, seuls $16 \%$ des foyers sont des familles et $50 \%$ des foyers sont des personnes isolées. Nous avons une société complètement différente si on compare avec les philosophies et les idées du logement de l'époque fordiste. Alors je ne dirais pas que nous sommes des révolutionnaires, nous sommes des réalistes. Nous essayons de comprendre ce qui se 
passe dans notre société, aussi dans les quartiers. Maintenant nous avons ces anciens quartiers énormes, coopératifs, où il n'y aucun commerce où il n'y a aucune possibilité de travailler. Dans les projets, on évite aujourd'hui cette séparation claire des fonctions. Les familles existent pour une vingtaine d'années, mais avant et après, ça change, et nous essayons de travailler avec cette fluidité de la vie post-moderne. En créant différentes possibilités de modes de vie, ce qui inclut aussi l'infrastructure du quartier, le boulot, le travail. Nous sommes dans une phase de développement où on passe du principe de créer des lotissements à créer des quartiers. Dans les nouveaux projets comme Mehr als wohnen, il y a vraiment une échelle urbanistique. Alors nous discutons des lieux où la ville peut accueillir des nouveaux nœuds d'intensité. Nous essayons de bouleverser le système existant de la ville en créant des structures très denses. Nous discutons des possibilités de reconfigurer la périphérie de la ville de Zurich. Aujourd'hui nous avons un tapis énorme. La moitié de mon pays est sur-urbanisé et il faut restructurer ça. Cela ne peut pas survivre dans une société post fossile, ça gaspille beaucoup trop d'énergie. Et ça n'est pas durable au niveau social parce que les gens sont seuls dans leur maison unifamiliale. II faut restructurer cette relation centrepériphérie.

Quel est le lien entre le modèle de coopératives de logement suisse et l'idée des communs ou l'idée du allmende en allemand?

Nous jouons un peu avec le mythe (rires). Si on se lie avec l'histoire des coopératives, et aussi avec I'histoire du pays, la Suisse porte dans son nom l'idée de coopérative : Schweizerische Eidgenossenschaft (confédération helvétique). Alors nous sommes, comme pays, une coopérative, nous jouons avec ces mots, mais naturellement il faut comprendre I'histoire : les mots sont les mêmes mais leur sémantique a changé. Alors ça a commencé au Moyen Âge. Nous n'avons jamais eu de rois ni de grands paysans. C'était difficile de survivre parce qu'il y avait beaucoup de montagnes. Ça a forcé les agriculteurs à s'organiser en coopérative. Les petits paysans pour faire cette transhumance, pour cultiver les Alpes, ont dû s'organiser. Et ils ont inventé des coopératives agricoles au Moyen Âge qui ont aussi organisé les terres. Alors les allmende, les communs en Suisse, ce n'étaient pas seulement des espaces dans les villages cultivés collectivement, mais c'étaient toutes les Alpes. II y a beaucoup de villages et de communautés en Suisse où $50 \%$ des terrains, encore aujourd'hui, sont la propriété des communaux. Ils appar- tiennent aux gens qui vivent dans cette commune. Alors il y a deux structures séparées. II y a une démocratie normale moderne, mais il y a aussi les coopératives des citoyens, qui sont maintenant des fondations ou des coopératives ou des associations qui possèdent aujourd'hui encore beaucoup de forêts, de terrains communs. Les communs en Suisse et les coopératives, ça vient du monde agricole. Elinor Ostrom a utilisé le modèle suisse comme contre-thèse de “ la tragédie des communs » pour montrer que ça peut fonctionner.

D'un point de vue spatial, comment les coopératives déclinent-elles l'idée de communs à différentes échelles ? En partant de l'échelle du logement jusqu'à celle de la ville, comment ces coopératives offrent-elles des espaces communs pour la collectivité ?

Pour comprendre ce que nous faisons au niveau spatial, il faut revenir aux aspects sociaux de ces projets. Si tu es dans la coopérative, tu es producteur et consommateur en même temps. Avec les parts sociales, tu es investisseur. Tu n'es pas dans ton propre appartement, mais dans toute la coopérative. Et en même temps, tu veux produire un projet qui puisse sauver ton problème d'habitation. Ce lien entre consommation et production, ça te met dans une situation complètement démocratique, et différente de celle des investisseurs privés, qui ont une certaine somme d'argent qu'ils veulent investir dans un lotissement. Ils calculent un certain loyer qui leur permet de payer leur intérêt et tout ça. Cette situation différente, ça produit tout ce que nous faisons, je dirais. Parce que dès l'initiation, la question est : «Qu'est-ce qu'on fait ? Qu'est-ce qu'on veut ? ". Ces questions sont au centre du projet. Alors ça permet de produire des projets très ouverts. C'est le commons comme projet, on pourrait dire. Mais le principe, c'est le système démocratique ouvert qui discute et finalement construit une intervention spatiale dans la ville. Et je crois que cette situation crée - parce que notre société est changeante - des expérimentations, de la recherche : comment nous voulons, comment nous pouvons nous organiser Parce que cette phase de la famille nucléaire dans son appartement avec une porte qui est une limite assez forte, c'est une phase qui représente une centaine d'années dans la vie de l'humanité, c'est une phase très courte qui a été produite par l'industrialisation. Je ne suis pas romantique mais je crois qu'aussi sur ce niveau nous essayons de repenser des structures beaucoup plus ouvertes où le travail et le logement sont beaucoup plus liés, où il y a des relations multigénérationnelles dans les foyers, où l'on vit avec des gens qui travaillent et d'autres 
non. Il faut vraiment être très précautionneux de ne pas devenir romantique sur les phase préindustrielles mais il y a des images avec lesquelles on peut travailler, on peut s'inspirer parce que personne ne sait comment on va travailler dans les décennies à venir quand le transport et la consommation seront réorganisés avec des relations entre des structures un peu plus locales, un peu plus cycliques : le recyclage, la production urbaine de nourriture par exemples, sont des sujets très importants en ce moment. Les gens s'occupent de la nourriture, ils veulent comprendre comment c'est fait. Ce sont des forces et des sources très importantes pour nos projets.

\section{Pourrais-tu nous expliquer comment tu habites aujourd'hui ?}

(Rires) J'ai beaucoup déménagé... une vingtaine de fois ! Ce n'était vraiment pas simple de trouver un logement dans ma ville si tu n'es pas riche, si tu es étudiant. Finalement on pourrait dire que j'ai construit tous ces projets pour moimême (rires). On a décidé dans le premier projet dans Kraftwerk 1 (centrale électrique) de construire des colocations énormes. C'était le premier bâtiment construit, nouveau, où on a créé des collocations. Ça existait dans les maisons existantes, mais pas dans un truc nouveau. Nous n'avons pas su comment louer ça. C'était des structures de 300 $\mathrm{m}^{2}$ avec onze chambres individuelles, mais on ne savait pas comment organiser la location de ces structures. Nous avons des chambres individuelles, nous partageons le reste, environ la moitié de l'appartement. Alors nous avons dit que tous les membres des équipes des directeurs de projet, dont je faisais partie seraient responsables d'une colocation. Nous avons réparti les cinq grandes colocations entre nous et donc j'ai habité là alors que je n'avais pas forcément cette idée à la base. Finalement, on a commencé par trouver des amis, à s'organiser. Maintenant, nous vivons à onze depuis seize ans. Je n'ai jamais vécu aussi longtemps dans un logement. Et ça fonctionne très bien.

Dans notre colocation, ce sont des habitants de 0 à 60 ans. Naturellement il $y$ a des gens qui viennent et des gens qui quittent, il y a des familles, il y a plusieurs enfants qui y sont nés. Nous avons des chambres individuelles, nous partageons le reste, environ la moitié de l'appartement : un grand salon que personne n'aurait pu avoir dans une structure conventionnelle, une grande cuisine où nous cuisinons ensemble, nous avons la nourriture, c'est surtout la nourriture et les repas qui créent le lien entre les gens.

Visionner la conférence en ligne https://youtu.be/CZXOadRhHmE

1ère édition du MOOC Récits d'urbanisme et question des communs : mars-juin 2018.

$2^{e}$ édition du MOOC Récits d'urbanisme et question des communs : septembre-décembre 2018.
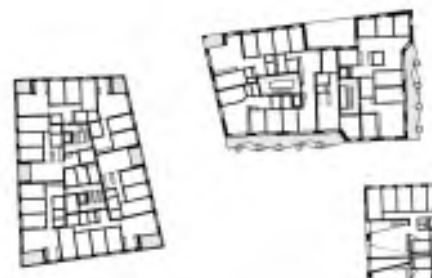

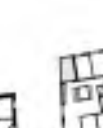
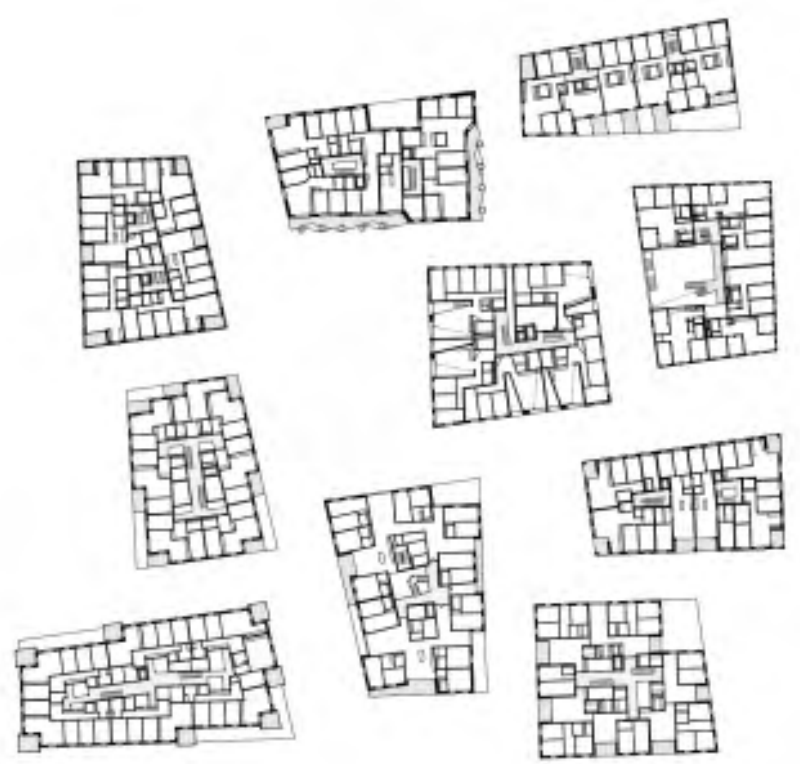

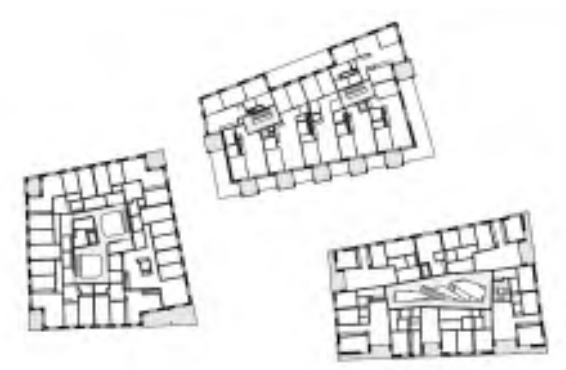

